\title{
15. \\ Ueber die Theorie der Schraube.
}

(Vom Herrn Prof. Dr. Lehmus zu Berlin.)

Gregen diese einfache Theorie, wie sie gewöhnlich vorgetragen wird, sind einige Erinnerungen zu machen. Der gewöhnliche Vortrag ist folgender:

Wenn auf einer schiefen Ebene, welche unter dem Winkel $\propto$ gegen den Horizont geneigt ist, ein Gewicht $Q$ liegt, die Kraft $P$ aber horizontal auf Erhebung von $Q$ wirkt, und $\mu$ den Reibungs-Coëfficienten bezeichnet, so ist

$$
P=\frac{\operatorname{tang} \alpha+\mu}{1-\mu \operatorname{tang} \alpha} \cdot Q \text {. }
$$

Wirkt nun bei einer Schraube mit lothrechter Spindel, deren Gewicht, nebst der daran hängenden Last, $=Q$ ist, die Kraft $R$ horizontal in der Entfernung $b$ von der lothrechten Achse, und bezeichnet $a$ den Winkel der Schrauben-Gänge gegen den Horizont, $r$ den mittleren Halbmęsser der Spindel und $h$ die Höhe jedes Schraubengangs, so wäre die in der Peripherie $2 r \pi$ tangential thätige Kraft $p$ nach obiger Formel:

oder

$$
=\frac{\operatorname{tang} \alpha+\mu}{1-\mu \operatorname{tang} \alpha} \cdot Q
$$

$$
p=\frac{h+\mu \cdot 2 r \pi}{2 r \pi-\mu h} \cdot Q,
$$

und folglich, wenn dieselbe auf den Hebelsarm $b$ reducirt wird:

$$
R=\frac{p r}{b}
$$

oder

$$
R=\frac{r}{b} \cdot \frac{h+\mu \cdot 2 r \pi}{2 r \pi-\mu h} . Q .
$$

Dieser Formel liegt aber, obgleich etwas versteckt, folgende, wie es scheint, irrige Ansicht zum Grunde.

$\therefore \quad$ Reducirt man nemlich die in der Entfernung $b$ erforderliche Kraft $R$ in die Entfernung $r$, so hat man $\frac{R b}{r}$, und aus dieser horizontalen Thätigkeit entspringen die Kräfte $\frac{R b}{r} \cos \alpha$ und $\frac{\dot{R} b}{r} \sin \alpha$, erstere parallel mit der 
Schraubenlinie, letztere normal darauf, und eben so zerlegt sich $Q$ in $Q \sin \alpha$ und $Q \cos \alpha$. Die Normal-Pressung, welche Reibung erzeugt, is daher $=\frac{R b}{r} \sin \alpha+Q \cos \alpha$, und die Bedingungsgleichung also

$$
\frac{R b}{r} \cos \alpha=Q \sin \alpha+\mu\left[\frac{R b}{r} \sin \alpha+Q \cos \alpha\right],
$$

woraus wie oben:

folgt.

$$
\boldsymbol{R}=\frac{r}{b} \cdot \frac{h+\mu \cdot 2 r \pi}{2 r \pi-\mu h} \cdot Q
$$

Das Unrichtige dieser Vorstellung besteht nun darin, dafs der aus $R$ entspringende, Reibung erzeugende Normaldruck $=\frac{R b}{r} \sin \alpha$ gesetzt wurde, obgleich derselbe nur der wirklich vorhandenen Kraft $R$, nicht der blofs thätig gedachten $\frac{R b}{r}$ entsprechen kann, atso nur die Gröfse $R \sin \alpha$ hat. Bringt man daher, wie gehörig, diese in Rechnung, so ist die Gleichung:

$$
\frac{R b}{r} \cos \alpha=Q \sin \alpha+\mu[R \sin \alpha+Q \cos \alpha],
$$

und hieraus folgt:

$$
\boldsymbol{R}=\frac{h+\mu \cdot 2 r \pi}{2 b \pi-\mu h} Q
$$

welches die genauere, auch in meinem Lehrbuch der angewandten Mathematik, 2ter Band, Berlin 1818, gegebene Formel ist.

Bequemer ist übrigens folgende Herleitung:

Die Kräfte, welche einander das Gleichgewicht halten sollen, sind:

$$
R ; Q \text { und } \mu[R \sin \alpha+Q \cos \alpha] \text {, }
$$

und die ihnen zugehörigen gleichzeitigen Wege:

folglich die Gleichung:

$$
2 b \pi ;-h \text { und }-2 r \pi \sec \alpha ;
$$

woraus sich ebenfalls

ergiebt.

$$
R .2 b \pi-Q h-\mu[R \sin \alpha+Q \cos \alpha] 2 r \pi \sec \alpha=0,
$$

$$
R=\frac{h+\mu \cdot 2 r \pi}{2 b \pi-\mu h} . Q
$$

